\title{
BEAMING CONE OF IO-CONTROLLED JOVIAN DECAMETER RADIO EMISSION AND EXISTENCE OF LOCALIZED ACTIVE LONGITUDE
}

\author{
P. H. M. Galopeau*, and M. Y. Boudjada ${ }^{\dagger}$
}

\begin{abstract}
The occurrence probability of the Jovian decameter radio emissions depends on two essential parameters: the central meridian longitude (CML) and the orbital phase of the satellite Io. Four main zones of enhanced occurrence probability emerge from the CML-Io phase diagram: the so-called Io-controlled sources Io-A, Io-B, Io$\mathrm{C}$ and Io-D. We study the compatibility of the location of these sources with the existence of a specific active longitude range, anchored in Jupiter's magnetic field, and favoring the radio emissions. A theoretical model, based on the cyclotron maser instability (CMI), was proposed a few years ago in order to explain the existence of such active longitudes, assuming that the radiation was emitted at the local gyrofrequency in a hollow cone of constant angle, along a magnetic field line carried away by Io through its revolution around Jupiter. Unfortunately this model was not able to justify the dimension in longitude of all the Io-controlled sources, in particular those located in the Jovian southern hemisphere (Io-C and Io-D). We show that the azimuthal distribution of the four occurrence regions (Io-A, Io-B, Io-C and Io-D) around the gradient of the local magnetic field is not constant so that the emission cone (in each Jovian hemisphere) presents a significant flattening in the direction of the magnetic field vector. Introducing a beaming cone with an elliptical section makes the location and extension in longitude of the sources (in the CML-Io phase diagram) compatible with the existence of an active longitude. A theory of the CMI, acting in an inhomogeneous medium in which the magnetic field vector and the gradient of its modulus are not aligned, shall be required in order to justify the flattening of the emission cone.
\end{abstract}

\section{Introduction}

Long term ground observations of the Jovian decameter radio emission show that the occurrence probability of the radiation depends on two essential parameters: the central

* LATMOS, Université Versailles Saint-Quentin-en-Yvelines, CNRS, 78280 Guyancourt, France

† Space Research Institute, Austrian Academy of Sciences, Schmiedlstrasse 6, A-8042 Graz, Austria 
meridian longitude (CML, System III) which is linked to the rotating magnetic field and the orbital phase of the satellite Io. The CML-Io phase diagram, which displays the occurrence of the emission as a function of the CML and the Io phase, reveals several zones of enhanced occurrence probability which have been named Io-controlled sources: Io-A, Io-B, Io-C and Io-D. Each source is a rectangle defined by a pair of CMLs and Io phases. For this study, we use the values given in Table 1.

Table 1: Limits of the rectangular source boxes

\begin{tabular}{|c|c|c|c|c|}
\hline Source & CML 1 & Io Phase 1 & CML 2 & Io Phase 2 \\
\hline A & $204^{\circ}$ & $180^{\circ}$ & $265^{\circ}$ & $254^{\circ}$ \\
B & $90^{\circ}$ & $66^{\circ}$ & $204^{\circ}$ & $110^{\circ}$ \\
C & $299^{\circ}$ & $221^{\circ}$ & $33^{\circ}$ & $254^{\circ}$ \\
D & $0^{\circ}$ & $90^{\circ}$ & $204^{\circ}$ & $110^{\circ}$ \\
\hline
\end{tabular}

We suppose that the radio emission is produced near the local gyrofrequency on active magnetic field lines which are carried out in Io's wake with a lead angle $\delta$. The emission is also supposed to be emitted in a hollow cone aligned with the gradient of the magnetic field modulus $-\nabla B$. The longitude of the active magnetic field line $\lambda_{a}$, the CML $\lambda_{\mathrm{CML}}$, the orbital phase of Io $\Phi_{\text {Io }}$ and $\delta$ are linked by the following equation:

$$
\lambda_{a}=\lambda_{\mathrm{CML}}+\pi-\Phi_{\mathrm{Io}}-\delta .
$$

In the present paper, we study how the location of the four Io-controlled sources Io-A, Io-B, Io-C and Io-D, in the CML-Io phase diagram, can put constraints on (i) the beaming cone of the radiation and (ii) the possible Jovian active longitude range. A theoretical calculation of a Jovian active longitude, followed by a parametric study and an analysis of observational constraints was done in previous papers [Galopeau et al., 2004, 2007; Galopeau and Boudjada, 2010].

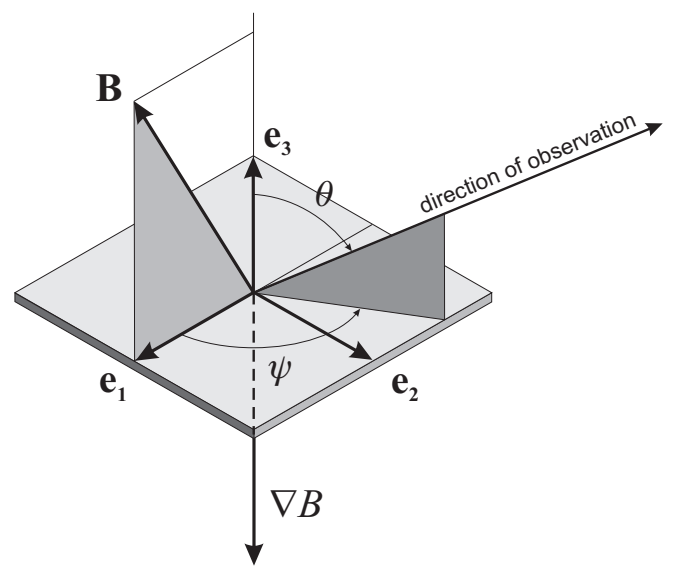

Figure 1: The local coordinate system is defined by the three vectors $\left(\mathbf{e}_{1}, \mathbf{e}_{2}, \mathbf{e}_{3}\right)$. The direction of observation is located by the two angles $(\theta, \psi)$. 

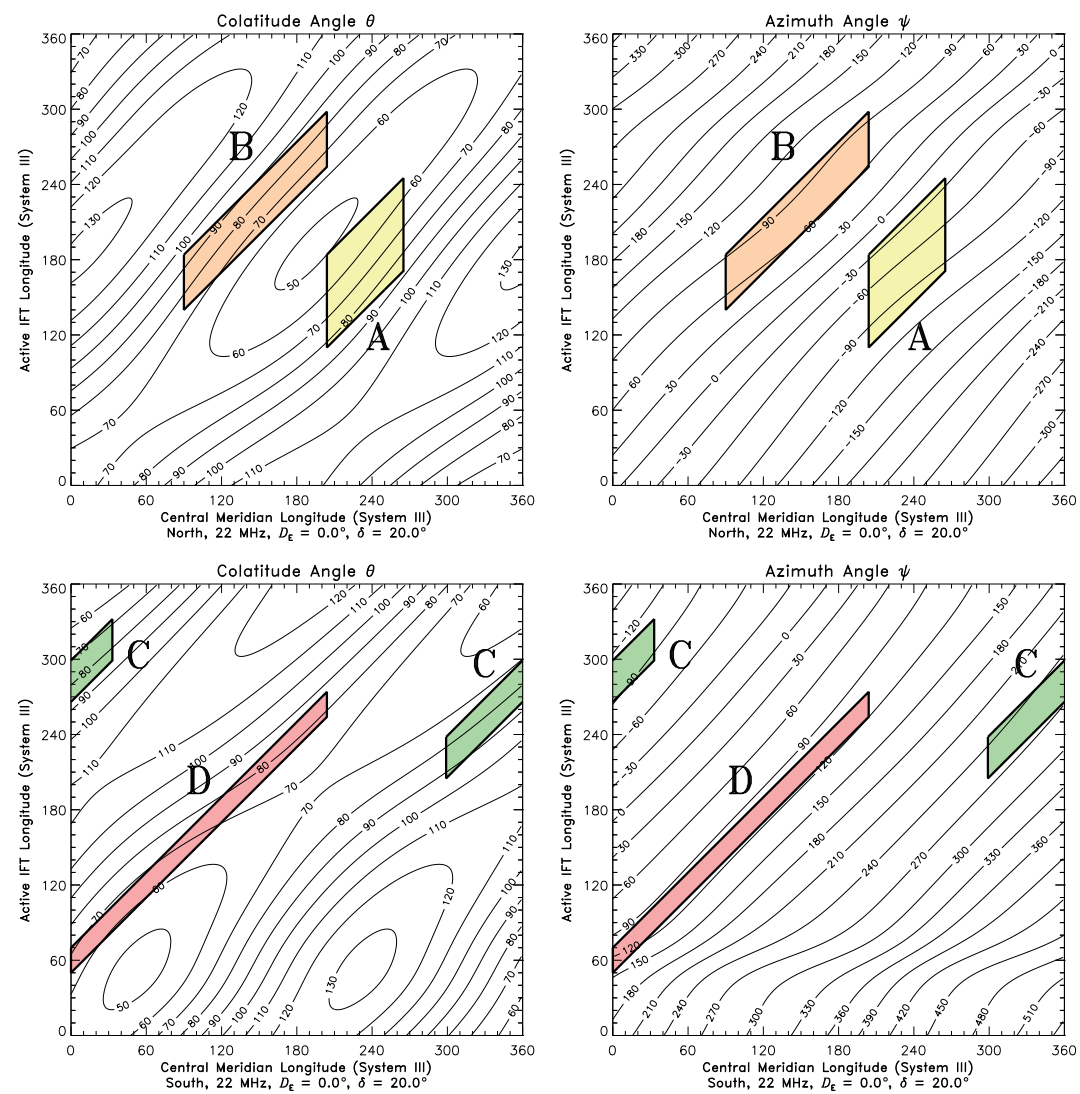

Figure 2: Contours of the colatitude angle $\theta$ (left panels) and the azimuth angle $\psi$ (right panels) as a function of the central meridian longitude (System III) and the longitude of the active Io flux tube for both hemispheres. The frequency is $f=22 \mathrm{MHz}$ and the lead angle $\delta=20^{\circ}$. The four boxes labelled $A, B, C, D$ correspond to the well-known Io-controlled decameter sources. The curves of constant $\theta$ correspond to the trace of axisymmetrical cones around $-\nabla B$.

\section{Polar Distribution of the Source Regions}

From the Jovian magnetic field vector $\mathbf{B}$ and the gradient of its modulus $\nabla B$ (derived from the O6 model), we define a local coordinate system (in the radio source) by the following three vectors:

$$
\mathbf{e}_{1}=\frac{\nabla B \times(\mathbf{B} \times \nabla B)}{\|\nabla B \times(\mathbf{B} \times \nabla B)\|}, \quad \mathbf{e}_{2}=\frac{\mathbf{B} \times \nabla B}{\|\mathbf{B} \times \nabla B\|}, \quad \mathbf{e}_{3}=-\frac{\nabla B}{\|\nabla B\|} .
$$

The vector $\mathbf{e}_{3}$ is parallel to $-\nabla B$ and $\mathbf{e}_{1}$ is oriented according to the projection of $\mathbf{B}$ onto the plane perpendicular to $\nabla B$. The direction of observation is located by the two angles 

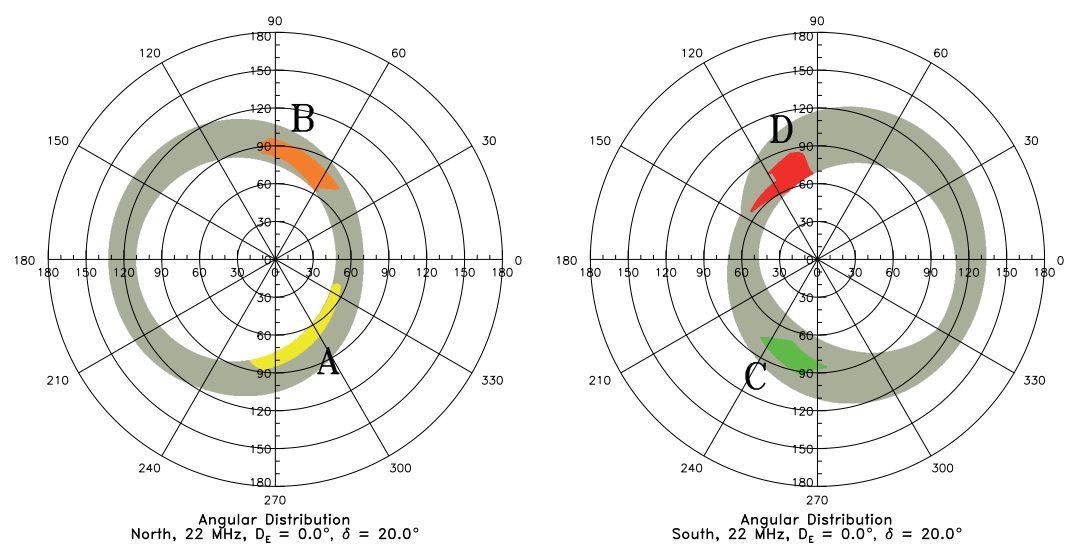

Figure 3: The four sources Io- $A$, Io-B, Io- $C$ and Io-D from Figure 2 are displayed in a polar diagram as a function of the colatitude angle $\theta$ (radial coordinate) and the azimuth angle $\psi$ for both hemispheres. The frequency is $f=22 \mathrm{MHz}$ and the lead angle $\delta=20^{\circ}$. The angular regions explored by the full CML-Io phase diagram are displayed in dark color.

$(\theta, \psi)$ where $\theta$ is the colatitude relatively to $\mathbf{e}_{3}$ and $\psi$ the azimuth the origin of which is $\mathbf{e}_{1}$ (see Figure 1). At the point of the magnetic field line where the gyrofrequency is $22 \mathrm{MHz}$, the angle between the directions of $\mathbf{B}$ and $-\nabla B$ is only a few degrees (less than $8^{\circ}$ ) whatever the active field line longitude may be.

Figure 2 displays the contours of the colatitude angle $\theta$ and the azimuth $\psi$ as a function of the central meridian longitude and the longitude of the active Io flux tube for the northern and southern hemispheres. The high occurrence probability regions for Io-A, Io-B, Io-C and Io-D are plotted in the same diagram. The angular distribution of these four sources is displayed in a polar diagram in Figure 3, revealing that the emission cone is not axisymmetrical around $-\nabla B$ but is flattened in the direction of $\mathbf{B}$. Figure 3 also suggests that the beaming of the radiation is distributed according to an ellipse the minor axis of which is located in the plane $(\mathbf{B}, \nabla B)$.

\section{Polar Distribution in Elliptic Coordinates}

We define a system of elliptic coordinates $(\xi, \eta)$ by the following equations:

$$
\begin{aligned}
\theta \cos \psi & =a \sinh \xi \cos \eta \\
\theta \sin \psi & =a \cosh \xi \sin \eta
\end{aligned}
$$

where $a$ is a constant scaling factor. Such a coordinate system is shown in Figure 4: the curves of constant $\xi$ are ellipses while the curves of constant $\eta$ are hyperbolae, all these lines share the same foci, separated by a distance equal to $2 a$. Thus, the elliptic beaming cone corresponds to an ellipse $\xi=\xi_{0}$. 
If $\theta_{\min }$ and $\theta_{\max }$ are the opening angles of the flattened emission cone respectively in the direction of the magnetic field and in the perpendicular direction, we can derive the values of $a$ and $\xi_{0}$ :

$$
\begin{aligned}
a & =\sqrt{\theta_{\max }^{2}-\theta_{\min }^{2}} \\
\xi_{0} & =\frac{1}{2} \ln \left[\frac{\theta_{\max }+\theta_{\min }}{\theta_{\max }-\theta_{\min }}\right] .
\end{aligned}
$$

The results are different for the two Jovian hemispheres. They are summarized in Table 2 and the corresponding ellipses are plotted in Figure 4.

Table 2: Opening angles of the flattened emission cone and corresponding values of $a$ and $\xi_{0}$.

\begin{tabular}{|c|c|c|c|c|}
\hline Hemisphere & $\theta_{\min }$ & $\theta_{\max }$ & $a$ & $\xi_{0}$ \\
\hline North & $43^{\circ}$ & $82^{\circ}$ & $69.8^{\circ}$ & 0.582 \\
South & $43^{\circ}$ & $89^{\circ}$ & $77.9^{\circ}$ & 0.527 \\
\hline
\end{tabular}

The contours of $\xi$ and $\eta$ versus the CML and active Io flux tube longitude are plotted in Figure 5. The trace of the flattened beaming cone (curve $\xi=\xi_{0}$ ) fits rather well the four source regions while it was not possible for an axisymmetrical cone of constant opening angle $\theta$ (see Figure 2).

The parametric equations of the emission cone, in the coordinate system defined by

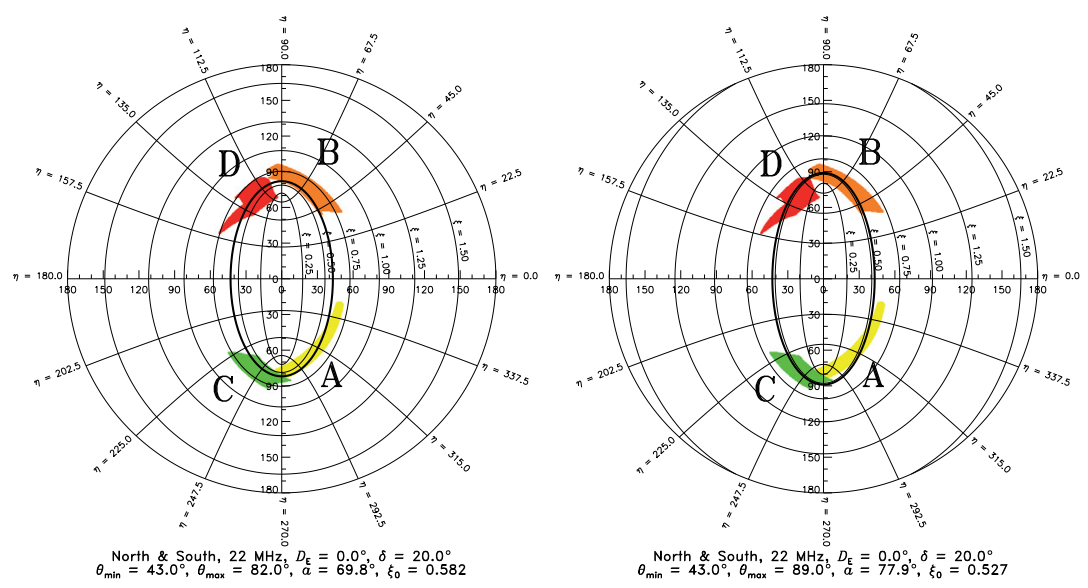

Figure 4: Polar diagram displaying the four Io-controlled sources and the elliptic coordinate lines which are confocal ellipses (curves of constant $\xi$ ) and hyperbolae (curves of constant $\eta$ ). The distance between the two foci is $2 a$. For each hemisphere, two numbers $a$ and $\xi_{0}$ are chosen so that the ellipse $\xi=\xi_{0}$ fits the source regions. The results are different for the northern sources (Io- $A$, Io- $B$, left panel) and southern ones (Io-C, Io-D, right panel). 

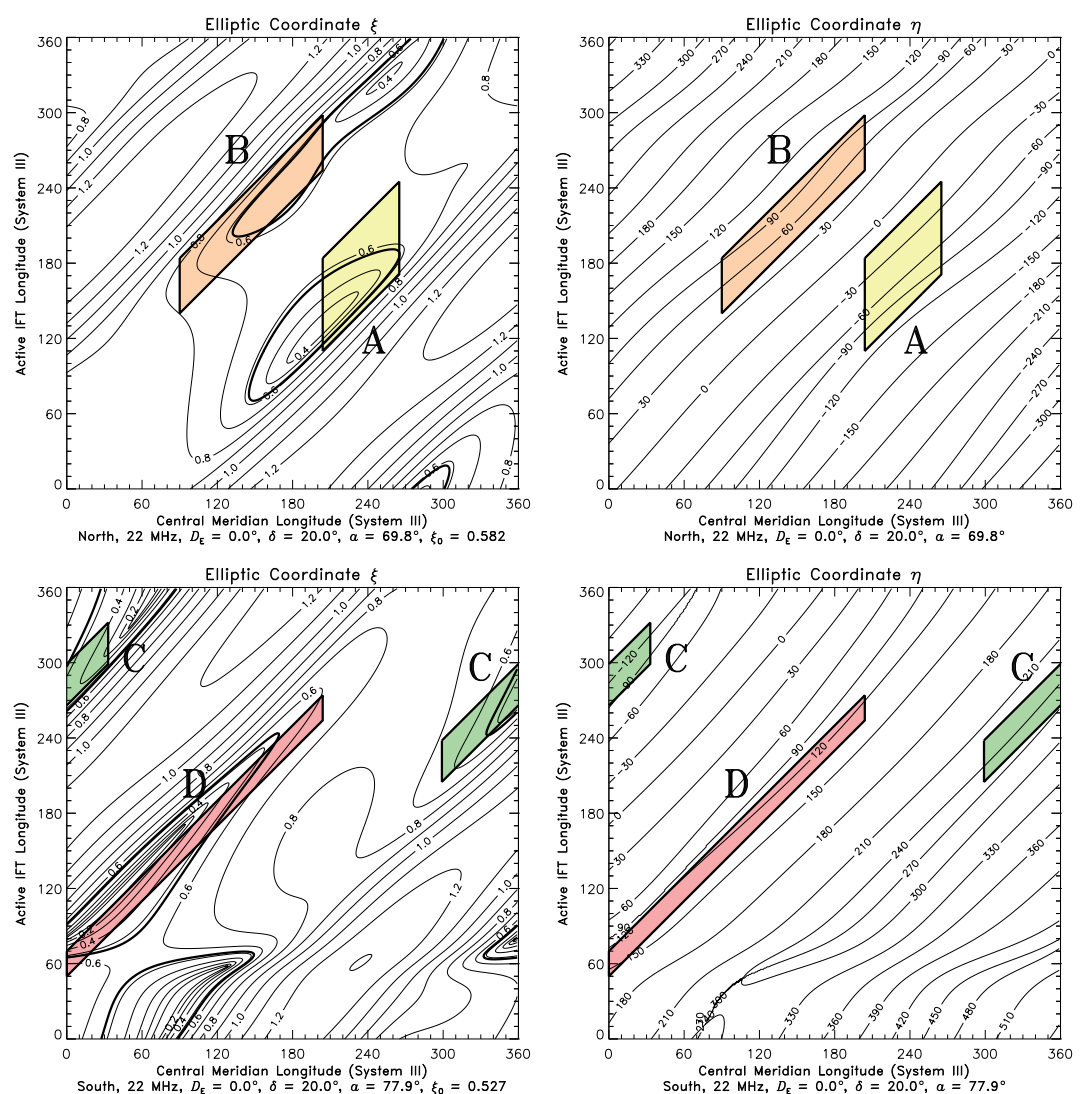

Figure 5: Contours of the elliptic coordinates $\xi$ (left panels) and $\eta$ (right panels) as a function of the central meridian longitude (System III) and the longitude of the active Io flux tube for the northern and southern hemispheres. The regions of high occurrence probability for Io- $A$, Io- $B$, Io- $C$ and Io-D are also displayed. The curve $\xi=\xi_{0}$ corresponds to the trace of the flattened beaming cone derived from Figure 4. The values of $a$ and $\xi_{0}$ are distinct for the two hemispheres.

$\left(\mathbf{e}_{1}, \mathbf{e}_{2}, \mathbf{e}_{3}\right)$, are:

$$
\begin{aligned}
& x=\sin \left[a \sqrt{\cosh ^{2} \xi_{0}-\cos ^{2} \eta}\right] \frac{\sinh \xi_{0} \cos \eta}{\sqrt{\cosh ^{2} \xi_{0}-\cos ^{2} \eta}} \\
& y=\sin \left[a \sqrt{\cosh ^{2} \xi_{0}-\cos ^{2} \eta}\right] \frac{\cosh \xi_{0} \sin \eta}{\sqrt{\cosh ^{2} \xi_{0}-\cos ^{2} \eta}} \\
& z=\cos \left[a \sqrt{\cosh ^{2} \xi_{0}-\cos ^{2} \eta}\right]
\end{aligned}
$$

with $\eta$ varying from $0^{\circ}$ to $360^{\circ}$. Two three-dimensional plots of the northern cone are 

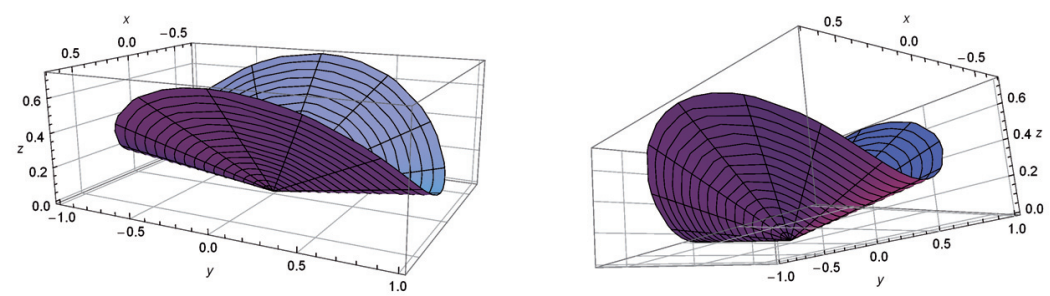

Figure 6: Three-dimensional plots of the northern flattened beaming cone derived from our study. The $z$-axis is parallel to $-\nabla B$ while the $x$-axis is in the direction of $\mathbf{B}$. The opening angle of the flattened cone is $\theta_{\min }=43^{\circ}$ in the direction of the magnetic field and $\theta_{\max }=82^{\circ}$ in the perpendicular direction ( $y$-axis).

shown in Figure 6.

The highest occurrence probability of the Jovian radio emission is reached when the beaming cone (described by the curve $\xi=\xi_{0}$ ) intersects a longitude range where the emission mechanism (supposed to be the cyclotron maser instability) is the most efficient. In Figure 7, such active longitude ranges have been chosen in order that the intersection corresponds as well as possible to the source regions.

As a result, a good fit of the source regions in the CML-Io phase diagram is obtained for: $150^{\circ} \leq \lambda_{a} \leq 270^{\circ}$ in the North, and $120^{\circ} \leq \lambda_{a} \leq 300^{\circ}$ in the South.

\section{Conclusions}

From our study, the location of the observed Io-controlled Jovian decameter sources in the CML-Io phase diagram allows us to put constraints on the emission cone and location of active longitudes. So we show that (i) the emission cone is not axisymmetrical around $-\nabla B$ but it flattened in the direction of the local magnetic field (the emission angle varies from $43^{\circ}$ up to $82^{\circ}$ or $89^{\circ}$ ), and that (ii) in each hemisphere, an active longitude range, favoring the radio emission and compatible with the observed sources, may exist $\left(150^{\circ}-270^{\circ}\right.$ in the North, $120^{\circ}-300^{\circ}$ in the South).

This complex three-dimensional geometry suggests a non-axisymmetrical amplification of the waves. Advanced theoretical studies concerning the propagation and the amplification of the radiation in inhomogeneous magnetospheric plasmas in the presence of both vectors $\mathbf{B}$ and $-\nabla B$ (with distinct directions) shall have to be done, in particular in the frame of the cyclotron maser instability. 

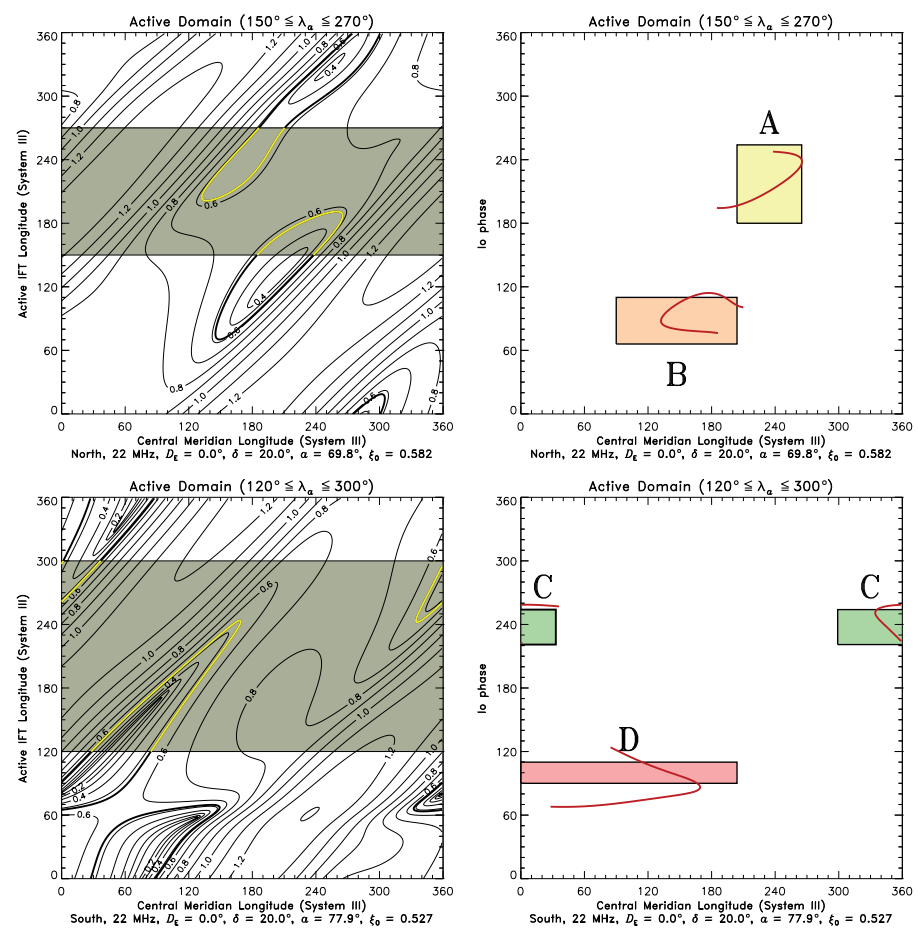

Figure 7: Left panels: Contours of the elliptic coordinate $\xi$ as a function of the central meridian longitude (System III) and the longitude of the active Io flux tube for the northern and southern hemispheres. A range of active longitude (displayed in dark color) has been chosen in order that the intersection (in yellow) with the flattened beaming cone $\left(\xi=\xi_{0}\right)$ corresponds to the regions of high occurrence probability. Right panels: The intersection of the active longitude domain with the flattened beaming cone is displayed versus central meridian longitude and Io phase (red curves). The observed Io-controlled sources Io- $A, I o-B$, Io-C and Io-D are also displayed.

\section{References}

Galopeau, P. H. M., M. Y. Boudjada, and H. O. Rucker, Evidence of Jovian active longitude: 1. Efficiency of cyclotron maser instability, J. Geophys. Res., 109, A12217, doi:10.1029/2004JA010459, 2004.

Galopeau, P.H. M., M. Y. Boudjada, and H. O. Rucker, Evidence of Jovian active longitude: 2. A parametric study, J. Geophys. Res., 112, A04211, doi:10.1029/ 2006JA011911, 2007.

Galopeau, P. H. M., and M. Y. Boudjada, Evidence of Jovian active longitude: 3. Observational constraints, J. Geophys. Res., 115, A12221, doi:10.1029/2010JA015677, 2010 . 